\section{IL-17A-Antikörper bei palmoplantarer Psoriasis}

\author{
Psoriasisbedingte Hautveränderungen an Händen und Füßen können jedes \\ Zugreifen und jeden Schritt zur Tortur werden lassen. An diesen Stellen ist der \\ Schuppenflechte allerdings nur schwer beizukommen. Laut einer aktuellen \\ Phase-III-Studie verspricht der humane Antikörper Secukinumab Linderung.
}

$\mathrm{D}$ ie palmoplantare Plaque-Psoriasis gilt gemäß der Definition der National Psoriasis Foundation als schwere Psoriasisform. Sie ist besonders hartnäckig und nur schwer zu behandeln. Zur primären systemischen Therapie der moderaten bis schweren Psoriasis bei Erwachsenen ist seit 2015 ein humaner monoklonaler Antikörper gegen Interleukin-17A, Secukinumab, zugelassen. Dessen Wirksamkeit speziell bei der palmoplantaren Psoriasis wurde nun randomisiert und doppelblind untersucht. An der industriegesponserten Phase-IIIStudie GESTURE arbeiteten Dermatologen aus 15 Ländern.

Insgesamt 205 Probanden mit palmoplantarer Plaque-Psoriasis wurden $\mathrm{zu}$ gleichen Teilen in drei Gruppen randomisiert und erhielten entweder den An- tikörper Secukinumab in einer Dosierung von $300 \mathrm{mg}$ oder in einer Dosierung von $150 \mathrm{mg}$ oder Placebo. In den ersten drei Wochen wurde das Medikament oder das Placebo einmal wöchentlich subkutan injiziert, von Woche vier bis Woche $16 \mathrm{im}$ Abstand von vier Wochen.

In der Auswertung berücksichtigt wurden 190 Patienten. Mehr als die Hälfte war systemisch vorbehandelt. Bereits Biologika eingenommen hatten nur $10,7 \%, 7,8 \%$ ohne den gewünschten Erfolg.

Nach 16 Wochen war bei $33,3 \%$ der mit 330 mg Secukinumab behandelten Probanden die Haut an den Handinnenflächen oder Fußsohlen frei oder fast frei (ppIGA 0/1) von psoriasisbedingten Hautveränderungen. Bei der 150-mg-
Dosierung waren es noch $22,1 \%$, in der Placebogruppe nur $1,5 \%$. Secukinumab zeigte sich somit in beiden Dosierungen wirksamer als Placebo (300 mg vs. Placebo: $\mathrm{p}<0,0001 ; 150 \mathrm{mg}$ vs. Placebo: $\mathrm{p}=$ $0,0002)$, wobei die höhere Dosierung durchweg effektiver war.

Über Nebenwirkungen berichteten $58 \%$ der Patienten der 300-mg-Gruppe, 64,7\% der 150-mg-Gruppe und 50,0\% der Placebogruppe. Am häufigsten wurden Kopfschmerzen, Nasopharyngitiden und Infektionen der oberen Atemwege genannt.

Fazit: Nach Ansicht der Studienautoren hat Secukinumab seine Wirksamkeit bei der Behandlung der palmoplantaren Psoriasis im Vergleich zu Placebo eindeutig unter Beweis gestellt. GESTURE stütze somit die Evidenz, dass Secukinumab als wirksame und sichere Therapieoption der palmoplanteren Psoriasis empfohlen werden kann.

Dr. Dagmar Kraus

Gottlieb A et al. Secukinumab shows significant efficacy in palmoplantar psoriasis: Results from GESTURE, a randomized controlled trial. J Am Acad Dermatol 2017; 76: 70-80

\section{Melanom: breites Spektrum an Nebenwirkungen}

\section{Anti-PD-1-Antikörper sind eine effektive Therapieoption bei metastasiertem Melanom und anderen Krebsarten. Allerdings können sie immunbedingte unerwünschte Ereignisse induzieren.}

mmunbedingte Nebenwirkungen der Therapie mit Anti-PD-1-Antikörpern können die Haut, den Gastrointestinaltrakt, die Leber, das Hormonsystem und andere Organsysteme betreffen. Sie können auch noch einige Zeit nach Therapiebeginn und möglicherweise sogar nach dem Absetzen der Medikamente auftreten. Eine adäquate Diagnose und das Management dieser Nebenwirkungen sind essenziell.

Um weitere Erkenntnisse zu diesen $\mathrm{Ne}$ benwirkungen $\mathrm{zu}$ erhalten, wurde eine retrospektive Studie an 15 Hautkrebszentren in Deutschland und der Schweiz durchgeführt. In die Studie wurden 496 Patienten mit metastasiertem Melanom aufgenommen, die mit Pembrolizumab oder Nivolumab behandelt wurden.
Im Rahmen der Studie wurden 242 Nebenwirkungen bei 138 Patienten beobachtet. Am häufigsten waren Hautreaktionen wie Juckreiz, Rash, Ekzem, Vitiligo, Alopezie, lichenoide und zytotoxische Reaktionen. Sie betrafen 8,7\% der Patienten. Nur drei Hautreaktionen wurden als Grad 3 klassifiziert, alle anderen waren in den Graden 1 - 2 und konnten, sofern erforderlich, mit topischen Kortikosteroiden oder systemischen Antihistaminika behandelt werden.

$4,2 \%$ der Patienten litten an gastrointestinalen Nebenwirkungen, darunter Diarrhö, Colitis, Bauchschmerzen, Koprostase, Xerostomie und Ösophagitis. Die meisten dieser Ereignisse waren leicht, es wurden nur vier Nebenwirkungen im Grad 3 beobachtet.
Eine Hepatitis trat bei 2,2\% der Patienten auf, wobei alle Ereignisse in den Graden 3-4 waren und mit Kortikosteroiden behandelt wurden.

Endokrine Erkrankungen wie Hypound Hyperthyreose, Hypophysitis, Nebenniereninsuffizienz und Diabetes wurden bei $6 \%$ der Patienten beobachtet. Etwa jedes vierte dieser Ereignisse war in den Graden 3-4.

Fazit: Anti-PD-1-Antikörper können vielfältige immunbedingte unerwünschte Ereignisse auslösen. Es ist wichtig, diese potenziellen Nebenwirkungen zu kennen, um das Management verbessern zu können. Die Therapie mit Anti-PD-1Antikörpern muss nur dann unterbrochen werden, wenn schwere Nebenwirkungen auftreten. Die Patienten sollten angewiesen werden, jegliche Symptome ihrem Arzt zu melden. Judith Neumaier

Hofmann L et al. Cutaneous, gastrointestinal, hepatic, endocrine, and renal side-effects of anti-PD-1 therapy. Eur J Cancer 2016; 60: 190-209 